\title{
Measurements of the Exerted Pressure by Pelvic Circumferential Compression Devices
}

\author{
Simon P. Knops ${ }^{1}$, Marcel P.J.M. van Riel ${ }^{2}$, Richard H.M. Goossens ${ }^{3}$, Esther M.M. van Lieshout ${ }^{*}, 1$, \\ Peter Patka ${ }^{1}$ and Inger B. Schipper ${ }^{\S, 1}$
}

\author{
${ }^{I}$ Department of Surgery-Traumatology and ${ }^{2}$ Neuroscience, Erasmus MC, University Medical Center Rotterdam, \\ Rotterdam, The Netherlands \\ ${ }^{3}$ Department of Industrial Design Engineering, section Applied Ergonomics and Design, Technical University Delft, \\ Delft, The Netherlands \\ ${ }^{\S}$ Current Address: Department of Surgery, Leiden University Medical Center, Leiden, The Netherlands
}

\begin{abstract}
Background: Data on the efficacy and safety of non-invasive Pelvic Circumferential Compression Devices (PCCDs) is limited. Tissue damage may occur if a continuous pressure on the skin exceeding $9.3 \mathrm{kPa}$ is sustained for more than two or three hours. The aim of this study was to gain insight into the pressure build-up at the interface, by measuring the PCCD-induced pressure when applying pulling forces to three different PCCDs (Pelvic Binder ${ }^{\mathbb{B}}$, SAMSling ${ }^{\circledR}$ and T-POD ${ }^{\circledR}$ ) in a simplified model.

Methods: The resulting exerted pressures were measured at four 'anatomical' locations (right, left, posterior and anterior) in a model using a pressure measurement system consisting of pressure cuffs.

Results: The exerted pressure varied substantially between the locations as well as between the PCCDs. Maximum pressures ranged from 18.9-23.3 $\mathrm{kPa}$ and from 19.2-27.5 $\mathrm{kPa}$ at the right location and left location, respectively. Pressures at the posterior location stayed below $18 \mathrm{kPa}$. At the anterior location pressures varied markedly between the different PCCDs.

Conclusion: The circumferential compression by the different PCCDs showed high pressures measured at the four locations using a simplified model. Difference in design and functional characteristics of the PCCDs resulted in different pressure build-up at the four locations. When following the manufacturer's instructions, the exerted pressure of all three PCCDs tested exceeded the tissue damaging level $(9.3 \mathrm{kPa})$. In case of prolonged use in a clinical situation this might put patients at risk for developing tissue damage.
\end{abstract}

Keywords: Pelvic circumferential compression device, PCCD, non-invasive, pressure measurement.

\section{INTRODUCTION}

Pelvic fractures are common injuries as a result of highenergy trauma. The incidence of these fractures is increasing [1]. Pelvic fracture often results in massive hemorrhage. The origin of the blood loss can be found in venous plexus lesion, arterial injury, and bleeding from fracture sites. Anatomic reduction and stabilization of pelvic fractures prevents blood loss by limiting bleeding from the fracture site and by reducing the pelvic volume. By reducing pelvic volume, bleeding can be stopped by mechanisms of tamponade, clotting or hemostasis [2]. External stabilization of the pelvis should reduce transfusion requirements and length of hospital stay, and has shown to improve survival in patients with unstable pelvic fractures [3]. In order to provide fast and easy reduction and stabilization, external pelvic compression is necessary. The biomechanical approach of

*Address correspondence to this author at the Department of SurgeryTraumatology, Erasmus MC, University Medical Center Rotterdam, P.O. Box 2040, 3000 CA Rotterdam, The Netherlands; Tel: +31-10-7031050; Fax:+31-10-7032396; E-mail: e.vanlieshout@erasmusmc.nl pelvic compression has resulted in an introduction of a noninvasive compression method: the pelvic circumferential compression device (PCCD). A PCCD is a belt that is wrapped around the fractured pelvis and tightened with the closing mechanism. Currently, the three most commonly used PCCDs (Pelvic Binder ${ }^{\mathbb{B}}$, T-POD ${ }^{\circledR}$ and SAM Sling ${ }^{\mathbb{B}}$ ) (Fig. 1A-C) are applied in pre-clinical and clinical situations for patients with pelvic fractures.

Pelvic circumferential compression is used in the prehospital phase and contributes to early non-invasive hemodynamical stabilization within the 'Golden hour' $[4,5]$. Advanced Trauma Life Support (ATLS) guidelines advise the use of a PCCD when an unstable pelvic fracture is suspected or diagnosed as a technique to stabilize the patient hemodynamically by reducing blood loss. The PCCDs provide circumferential compression to the bones within the pelvis. Compression forces will be most pronounced at the area of the pelvic bones that lie closely underneath the PCCD. In a clinical setting this denotes bony landmarks like the sacrum and the greater trochanters [6]. Also, the exact application location of the PCCD strongly affects the local 


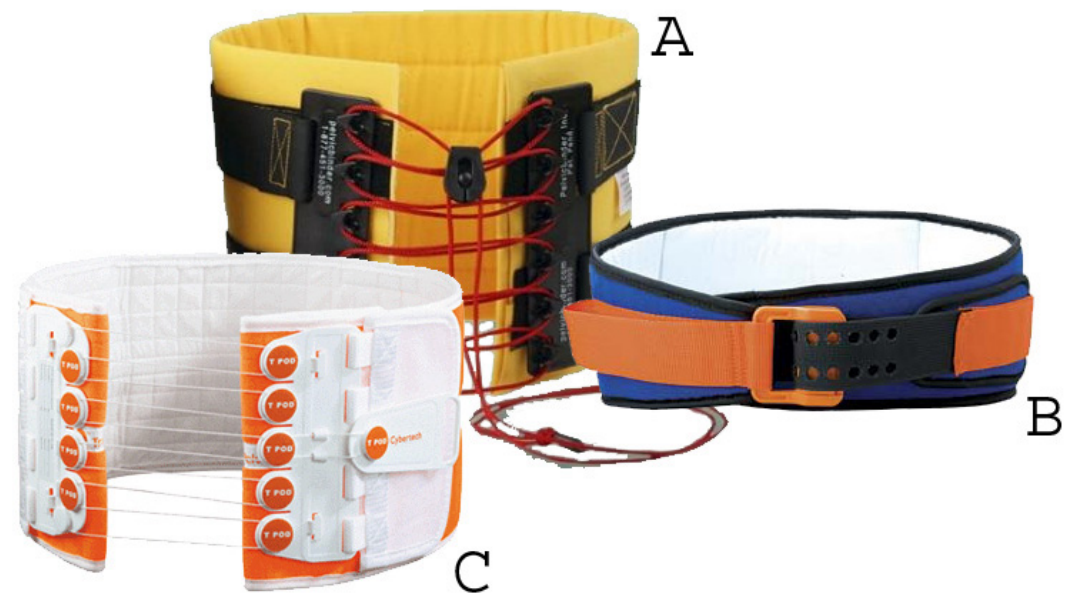

Fig. (1A-C). The three most frequently used PCCDs tested in this study.

1A Pelvic Binder ${ }^{\circledR}$

Manufacturer:

Size:

Closing mechanism:

Application instructions:

1B SAM-Sling ${ }^{\circledR}$

Manufacturer:

Size:

Closing mechanism:

Application instructions:

Pelvic Binder Inc., Dallas, TX, USA

One size fits all. "Cut-to-fit" 6-8" gap

Velcro-backed fastener with shoelace mechanism

Health care providers should be able to get at least two fingers between the patient and the binder after applying pressure

SAM Medical Products, Newport, OR, USA

Sized to fit. Extra Small, Standard, and Extra Large. Small belt leaving more space for clinical diagnostics or entrance to the abdomen

Fastener with an auto-stop buckle (33lbs) that limits circumferential compression at the time of PCCD application to the minimal required pulling force for pelvic reduction

Buckle placed close to midline. Pulled tight with or without assistance with two hands in opposite directions

\section{C Trauma Pelvic Orthodic Device ${ }^{\circledR}$ (T-POD)}

Manufacturer:

Size:

Closing mechanism:

Application instructions:
Bio Cybernetics Inter-national, La Verne, CA, USA

One size fits all. "Cut-to-fit" 6-8" gap.

Simultaneous circumferential compression through Velcro-backed mechanical advantage pulley system with a pull-tab

Health care providers should be able to insert two fingers between the patient and the T-POD pressure level and overall effects. Research into the transverse application level of a specific sling showed that the sling should be applied at the area of the greater trochanters and the symphysis pubis [7].

Data on the efficacy and safety of PCCDs is limited. The PCCDs currently available differ in design (material, shape and size) and closing mechanism, and may therefore have different functional characteristics, resulting in different mechanical and clinical effects. The applied pulling forces according to manufacturers application instructions differ substantially. A study on human cadaveric specimens showed the minimum strap tension required to achieve complete reduction of symphysis diastasis was $177 \pm 44 \mathrm{~N}$ and $180 \pm 50 \mathrm{~N}$ in the partially stable and unstable pelvis, respectively [7]. As opposed to the tension required to achieve complete reduction, there is no data available for the required tension to achieve hemostasis for initial resuscitation.
The forces that can be applied to the pelvic ring by the PCCDs are uncontrolled and unrestricted, except for the SAM-Sling ${ }^{\circledR}$, which has a fastener with an auto-stop buckle that limits circumferential compression when exceeding 150 $\mathrm{N}$ tensional force. The exerted pressure after applying a pulling force to the PCCD and the resulting effect on the underlying skin is unclear.

Adverse effects are related to high pressures on the skin and long-term use of the PCCD. The compression devices may cause pressure induced skin breakdown and accompanying co-morbidity in case of prolonged use in the period before invasive pelvic fixation. Tissue damage may occur if a continuous pressure exceeding $9.3 \mathrm{kPa}(9300$ $\mathrm{N} / \mathrm{m}^{2}$, corresponding with $69.8 \mathrm{mmHg}$ ) is sustained for more than two or three hours [8]. It is recommended that the pressure at the interface is kept below $4.66 \mathrm{kPa}$, i.e. below capillary blood pressure, allowing circulation to the skin to be maintained $[6,8]$. 
The aim of this study was to gain insight into the pressure build-up at the interface, by measuring the PCCD-induced pressure when applying pulling forces to three different PCCDs (Pelvic Binder ${ }^{\circledR}$, SAM-Sling ${ }^{\circledR}$ and T-POD ${ }^{\circledR}$ ) in a simplified artificial model.

\section{MATERIALS AND METHODOLOGY}

\section{Study Design}

The model used in this study was chosen to measure the pressure build-up without taking into account the anatomy of the human pelvis and should be considered as a simplified artificial model of a human pelvis. The model (Fig. 2) consisted of a cylindrical paper roll, with a height of $36 \mathrm{~cm}$ and a diameter of $38 \mathrm{~cm}$, wrapped in plastic foil. The model was placed upright in order to measure the exerted pressure produced by the PCCD, exclusively, without the weight of the model pressurizing as well.

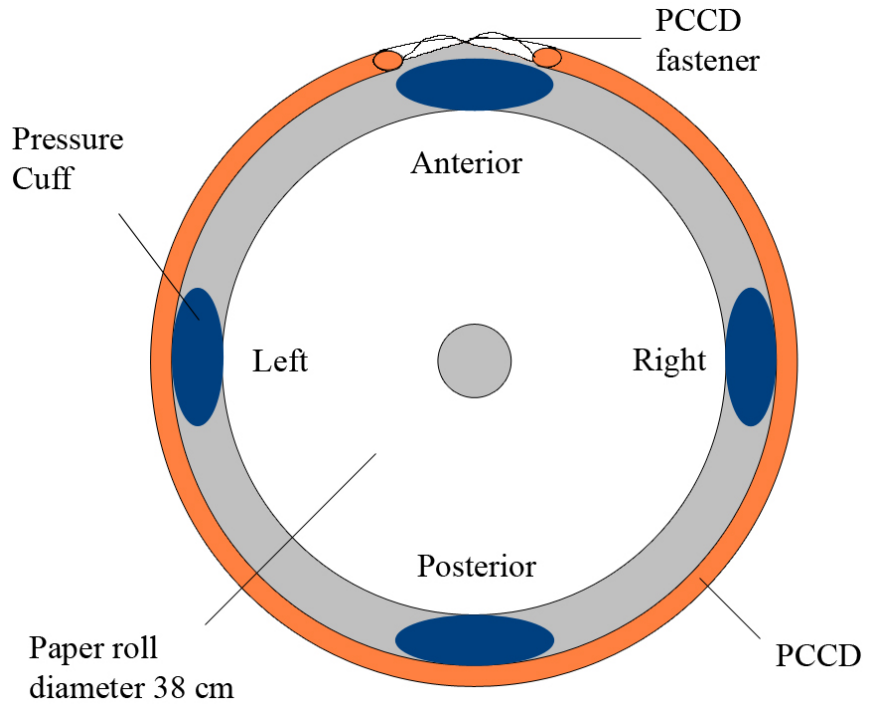

Fig. (2). Schematic representation of the measurement setup. A top view of the simplified artificial model of a pelvis consisting of a cylindrical paper roll, with a height of $36 \mathrm{~cm}$ and a diameter of 38 $\mathrm{cm}$, wrapped in plastic foil. The four air-filled oscillometric pressure cuffs were positioned in vertical position to the outside of the roll at the right, left, posterior and anterior locations. A PCCD covers the cuffs in the middle of the width of the PCCD with the fastener over the anterior location.

The commercially available PCCDs that were used are the Pelvic Binder ${ }^{\circledR}$, SAM-Sling ${ }^{\circledR}$ and the T-POD ${ }^{\circledR}$ (Fig. 1AC). All three PCCDs are disposable, radiolucent and MRI safe.

In these pressure measurements the smallest size noninvasive oscillometric pressure cuffs (Hewlett-Packard ${ }^{\mathrm{TM}}$, $\# 1$, limb circumference $3.1-5.7 \mathrm{~cm}$ ) were used. These cuffs are the same as cuffs used for measuring blood pressure in newborn infants.

An identification mark was pointed out at 0 degrees on the outside of the roll to mark the posterior location, representing the 'anatomical' location of the sacrum. Subsequently three marks were determined at 90, 180 and 270 degrees to mark the left, the anterior and the right location, representing estimated 'anatomical' locations of the left GT, the symphysis pubis, and right GT, respectively. Four pressure cuffs were attached in vertical position to the outside of the roll with tape. They were placed in line with the marks, with the tubes directed upward. The pressure cuffs were attached to the model of the pelvis and not directly to the binders to measure the pressure at the locations given. A schematic representation of the measurement setup is shown in Fig. (2).

Disposable transducers (DTXPlus $^{\mathrm{TM}}$ Pressure Transducer, PRESS VENEUS, REF 686495, Becton Dickinson, Franklin Lakes, NJ, USA) were attached to the pressure cuffs. The pressure cuffs were pressurized manually up to $6.7 \mathrm{kPa}(50 \mathrm{mmHg})$ before use and the pressure system was calibrated with a manometer. This pressure level was chosen to prevent collapse of the pressure cuffs. Measurements were recorded and displayed with use of the Multiple Channel Registration (MKR) digital data acquisitioning system (version 3.3.1, Directie Informatie, Erasmus MC, Rotterdam, The Netherlands). The resolution of this pressure system is $0.13 \mathrm{kPa}(1 \mathrm{mmHg})$. Each of the four pressure cuffs was connected separately to amplifier units that were connected to an ADC-board (AnalogueDigital Converter) in a personal computer.

\section{Description of Application of the PCCDs}

The PCCDs were tensioned with a stepwise $(20 \mathrm{~N}$ per step) increased pulling force until the maximum pulling force as mentioned in the manufacturer instructions was reached (Fig. 3). Except the SAM-Sling ${ }^{\circledR}$, which has a fastener with an auto-stop buckle that limits the pulling force to $150 \mathrm{~N}$. Pulling forces were consecutively increased every five seconds. The force applied to the binders was measured using a digital force gauge (9000 series CPU, AIKOH Engineering corp.) This gauge was connected to a separate amplifier unit. Measurements were performed in 'track' modus, implying continuous measurement.

The PCCDs were applied in a way that the cuffs were covered in the middle of the width of the PCCDs. The application instructions of the manufacturers were followed (Fig. 1). The application instructions did not consider the direction in which the PCCDs should be pulled tight. In order to measure in a standardised way the PCCDs were pulled tight with a horizontal pulling force at the tangens of the closing mechanism. The Pelvic Binder ${ }^{\circledR}$ was pulled tight downwards in the model with one hand, the SAM-Sling ${ }^{\mathbb{B}}$ was pulled tight with two hands in opposite directions and the T-POD ${ }^{\circledR}$ was pulled tight towards the right-hand side with one hand.

\section{Measurement Series}

The PCCDs were applied around the model and pulled tight and loosened three times before the measurements started. For each PCCD triplicate measurements were performed on day one. The four pressure cuffs were removed, switched and replaced at the same four locations on the model on day two. A second series of identical measurements was performed on day two. Each series consisted of four pressure signals measured at the four locations under the PCCD and the simultaneous recorded pulling force signal. The MKR digital data acquisitioning system was designed to process, plot and store the pulling 


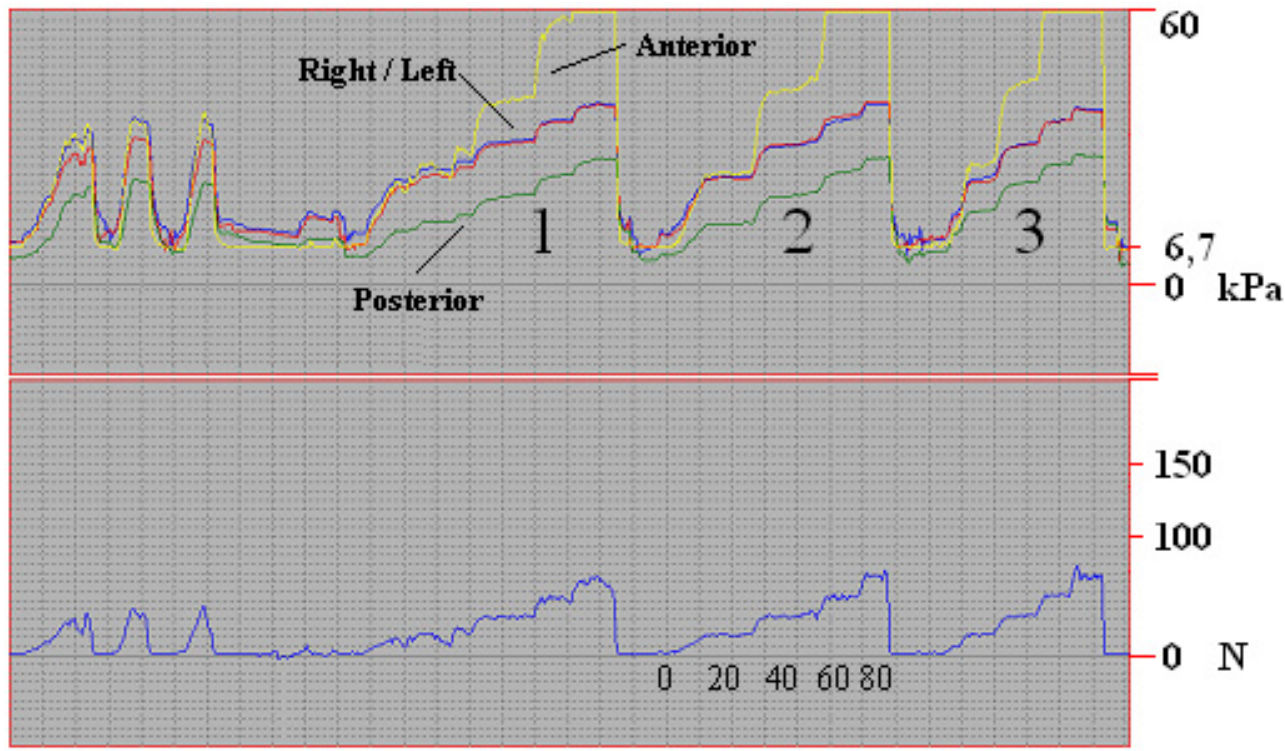

Fig. (3). A print from the MKR digital data acquisitioning system. Graphic representation of the exerted pressure (kPa) on the cuffs (upper panel) at four locations upon application of stepwise (20N per step) increasing pulling forces (N) to the PCCD (lower panel). 1, 2, and 3 represent effects of triplicate measurements of tightening and subsequent release of a PCCD. In each case, the pressure dropped to baseline upon release of the PCCDs.

force (one channel) and the resulting pressures (four channels), separately in two panels (Fig. 3).

\section{Statistical Analysis}

The means of all signals were calculated with MKR viewer (version 3.3.1., Direction Information, Erasmus MC) over manually selected intervals of approximately three seconds at sequenced levels of gradually increased pulling force of $20 \mathrm{~N}$ per step. For each interval the applied gauge force and the difference in pressure were corrected for baseline values. The data was further processed using GraphPad Prism, version 4.00.

\section{RESULTS}

The non-invasive pressure cuffs behaved elastically. Upon fastening and releasing the PCCDs, the cuffs always returned to their original state and calibrated pressure. A stepwise increase in tension resulted in a stepwise increase in exerted pressure (Fig. 3). The mean exerted pressure series on day one and day two are illustrated (Fig. 4). All twelve bar graphs show a linear trend for the exerted mean pressure on day one and two. The exerted pressure varies substantially, both between the different locations as well as between the different PCCDs. In order to calculate the average maximum pressure when strictly following the application instructions of the manufacturers, data of the six replicates were combined. The average maximum exerted pressure on the right location is $23.3 \mathrm{kPa}$ for the Pelvic Binder $^{\circledR}, 18.9 \mathrm{kPa}$ for the SAM-Sling ${ }^{\circledR}$ and $19.9 \mathrm{kPa}$ for the $\mathrm{T}-P O D^{\circledR}$. Likewise, the average maximum pressure is 21.3 $\mathrm{kPa}, 27.5 \mathrm{kPa}$ and $19.2 \mathrm{kPa}$ at the left location, $18 \mathrm{kPa}, 11.5$ $\mathrm{kPa}$ and $15.3 \mathrm{kPa}$ at the posterior location and $2.7 \mathrm{kPa}, 18.4$ $\mathrm{kPa}$, and $51.2 \mathrm{kPa}$ at the anterior location, respectively.

An exerted pressure of $10 \mathrm{kPa}$ is achieved with the Pelvic Binder $^{\circledR}$ and the T-POD ${ }^{\circledR}$ when a pulling force of $20 \mathrm{~N}$ is applied. For the SAM-Sling ${ }^{\circledR}$, on the other hand, a pulling force up to $40 \mathrm{~N}$ must be applied before the exerted pressure reaches $10 \mathrm{kPa}$.

An apparent observation is that measurements using the SAM-Sling ${ }^{\circledR}$ structurally produced higher mean pressures on the left location as compared to the right location. The measured exerted pressure on day one was markedly higher than the exerted pressure on day two.

With all three different PCCDs the exerted pressure on the posterior location was generally lower than the pressure measured at the right and left location. At the anterior location, the pressure exerted by the different PCCDs varied. Pressures with the Pelvic Binder ${ }^{\circledR}$ did not exceed $4 \mathrm{kPa}$, whereas with the T-POD ${ }^{\circledR}$ the pressure reached values that exceeded $52 \mathrm{kPa}$ (Fig. 4).

\section{DISCUSSION}

The measurements in this study provided insight into the pressure build-up by the three different PCCDs at the interface at four locations when using a novel simplified, artificial model of the human pelvis. Since the shape of the model does not reflect the anatomy of the human pelvis, extrapolation of the data to the human situation should be done with great caution.

Knowing the pulling force at which the pressure exerted by PCCDs exceeds the tissue damaging level $(9.3 \mathrm{kPa})$ is relevant from a clinical point of view, as that will indicate the risk of developing soft tissue damage upon prolonged use.

The finding that pressures returned to baseline upon release of the PCCDs implies that hysteresis (a property of systems that do not return completely to their original state, depending on its immediate history) has not affected these measurements. This is perceptible in Fig. (3). The linear trend between mean exerted pressure and mean force was expected. The finding that mean pressures of both days were 
Right
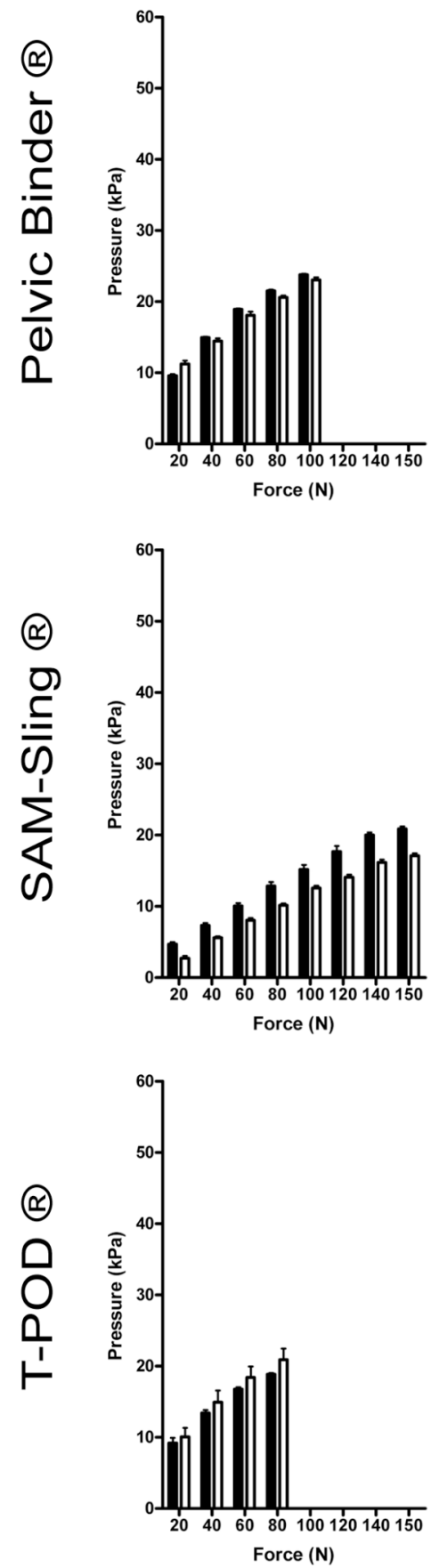

Left
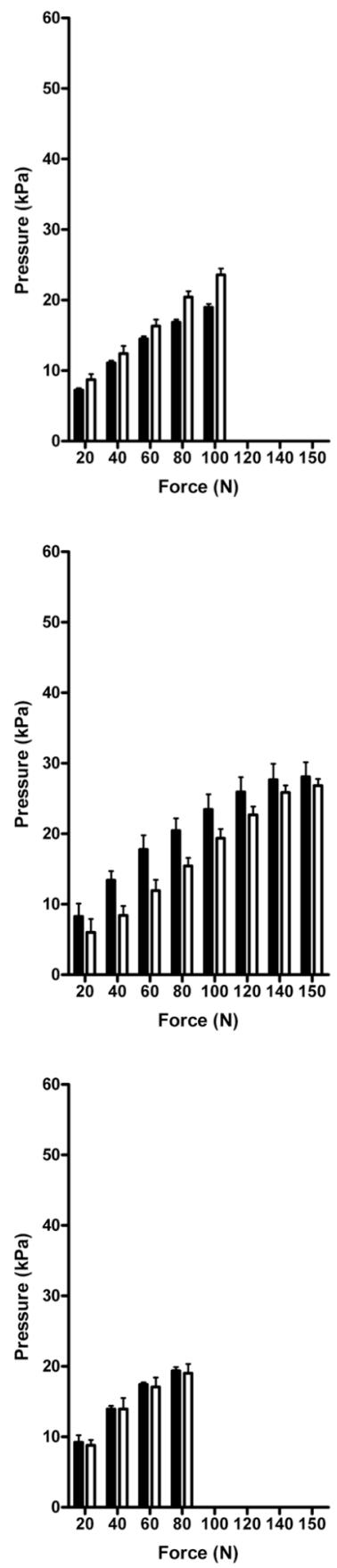

Posterior
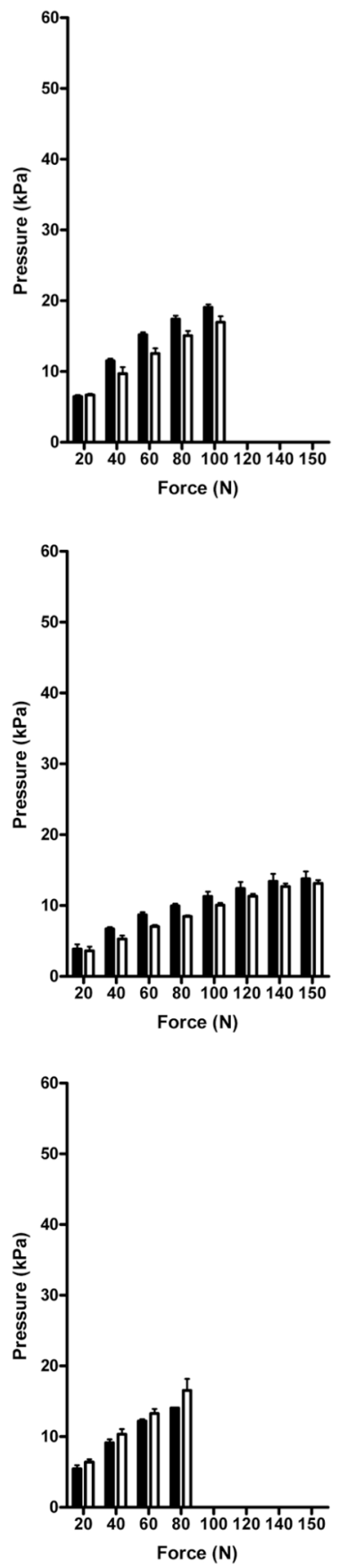

Anterior
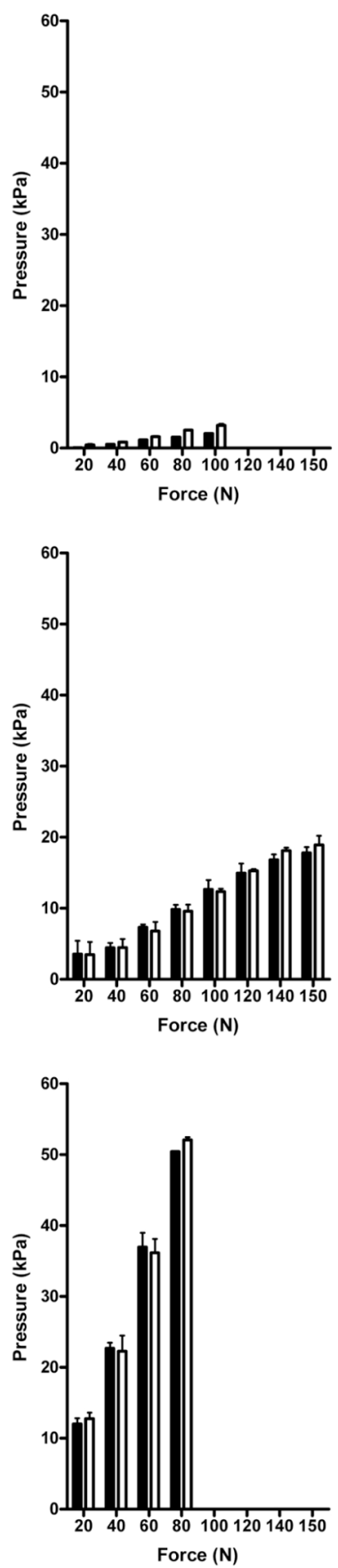

Fig. (4). Exerted pressure by three different PCCDs at the right, left, posterior and anterior locations. For each PCCD triplicate measurements were performed on two consecutive days. The mean exerted pressure series on day one (black bars) and day two (white bars) are illustrated. For each PCCD the error values: mean pressure and the Standard Error of the Mean (SEM) are represented. The y-axis scale shows the mean exerted pressure $(\mathrm{kPa})$ from 0 to $60 \mathrm{kPa}$. The $\mathrm{x}$-axis shows the mean pulling force $(\mathrm{N})$ with a categorical $20 \mathrm{~N}$ interval and ends with $150 \mathrm{~N}$. This categorical deviance at $150 \mathrm{~N}$ is due to the SAM-Slings auto-stop buckle. Measurements were performed according to manufacturer application instructions.

most alike for the T-POD ${ }^{\circledR}$ might imply that reproducibility of the measurements was best when using the T-POD ${ }^{\mathbb{R}}$. Measurements with the SAM-Sling ${ }^{\circledR}$ were subjected to the highest "between-day" variations. Measurements with the SAM-Sling ${ }^{\circledR}$ showed the lowest pressure build-up at the right and posterior locations and measurements with the T-POD ${ }^{\circledR}$ showed the lowest pressure build-up at the left location.
The most important differences between the binders are the closing mechanisms or fasteners and the size of the slings (Fig. 1A-C). The Pelvic Binder ${ }^{\circledR}$ has a shoelace mechanism which is liable to friction, causes a wedge shaped tightened binder, and results in increased pulling force. The SAMSling ${ }^{\circledR}$ has an auto-stop buckle (limited at $150 \mathrm{~N}$ ) and needs to be pulled together with two hands in opposite direction. 
The sling is relatively small as opposed to the other two PCCDs. It has the advantage of leaving more space for clinical diagnostics or entrance to the abdomen and the disadvantage of early misplacement at the level of the trochanters. The T-POD ${ }^{\circledR}$ has a mechanical advantage pulley system and only a small amount of pulling force is needed to accomplish simultaneous circumferential compression.

A quantification of the exerted pressure in the model showed that, when a standardized pulling force was put upon the PCCDs using the gauge, the pressure varied at the different locations underneath the PCCDs. There was a markedly difference between the different types of PCCD. Some of this variation is caused by the differences in the designs and the closing mechanisms of the PCCDs. Right location and left location mean pressure differences can be explained for the SAM-Sling ${ }^{\circledR}$ and the T-POD ${ }^{\circledR}$ as follows. The Velcro-belt of the SAM-Sling ${ }^{\circledR}$ is pulled tight through the auto-stop buckle with two hands in opposite directions, but most of the force is provided towards the right-hand side (left location), while facing the model. The pulling force first encounters the left location, thereby exerting a higher pressure on the left location than on the opposite right location. In contrast, the design of the T-POD ${ }^{\mathbb{R}}$ accounts for slightly higher mean pressures on the right location because this device is pulled tight in the opposite direction; towards the right-hand side in a straight line, while facing the model. The T-POD ${ }^{\circledR}$ is pulled tight against the substratum, the right location.

Because the measurements have been performed with the model in an upright position, the exerted pressure at the posterior location is due to the pressure delivered by the binder, and not by the gravitation. The exerted pressure on the posterior location in the current model is generally lower than the pressure measured at the right and left locations. A reason for this is that the posterior reference point is the farthest point from the fastener. The pulling force is not completely proportional passed on both right and left locations because of opposing friction forces. In clinical practice, the pressure at the posterior location will probably be higher, as patients will be in a supine position. In such situations, the resulting pressure at the posterior location (i.e. posterior pelvic region and the sacrum) will be a combination of the bodyweight of the patient and the pressure exerted by the binder. As a consequence, it is to be expected that in clinical situations the posterior pressure will be the highest of all four pelvic locations.

The mean pressure at the anterior location was difficult to assess and not reproducible for day one and two, although repeatability of multiple measurements on the same day showed only marginal differences in reproducibility. Major differences in closing mechanisms (i.e., shoelace versus auto-stop buckle versus pulley system) may be an explanation for these differences in exerted pressure. The exerted pressure with the T-POD ${ }^{\circledR}$ at the anterior location is very high as the fastener strings were cutting in the pressure cuff. In general, the data for the T-POD ${ }^{\mathbb{R}}$ show that the anterior location could form a risk-bearing area for the development of vascular insufficiency to skin tissue, especially in adipose persons (enlarged circumference with protrusive tissue). Placing a cover beneath the closing mechanism to protect the substratum might be a recommendable improvement.

\section{CONCLUSION}

The circumferential compression by the different PCCDs showed high pressures measured at the four locations using a simplified artificial model. Difference in design and functional characteristics of the PCCDs resulted in different pressure build-up at the four locations. When following the manufacturer's instructions, the exerted pressure of all three PCCDs tested exceeded the tissue damaging level $(9.3 \mathrm{kPa})$ in case of prolonged use. If these results were to be carefully extrapolated to a clinical setting, all three binders would cause a risk for skin problems, with regard to the exerted pressure.

Clinical research is necessary in order to measure the exerted pressure and resulting reduction characteristics of the different PCCDs in vivo. This may contribute to optimizing the application protocol of the current PCCDs for patients with pelvic fractures, and could also aid in the development of effective and safe PCCDs.

\section{REFERENCES}

[1] Pohlemann T. Pelvic ring injuries: Assessment and concepts of surgical management. Ruedi TP MW, ec. AO principles of fracture management. Stuttgart: Thieme Publishing Group 2000 2000:391413.

[2] Ghaemmaghami V, Sperry J, Gunst M, et al. Effects of early use of external pelvic compression on transfusion requirements and mortality in pelvic fractures. Am J Surg 2007; 194(6): 720-23.

[3] Croce MA, Magnotti LJ, Savage SA, Wood GW 2nd, Fabian TC. Emergent pelvic fixation in patients with exsanguinating pelvic fractures. J Am Coll Surg 2007; 204(5): 935-9; discussion 940-2.

[4] Nunn T, Cosker TD, Bose D, Pallister I. Immediate application of improvised pelvic binder as first step in extended resuscitation from life-threatening hypovolaemic shock in conscious patients with unstable pelvic injuries. Injury 2007; 38(1): 125-8.

[5] Meighan A, Gregori A, Kelly M, MacKay G. Pelvic fractures: The golden hour. Injury 1998; 29(3): 211-3.

[6] Vohra RK, McCollum CN. Pressure sores. BMJ 1994; 309(6958): 853-7.

[7] Bottlang M, Simpson T, Sigg J, Krieg JC, Madey SM, Long WB. Noninvasive reduction of open-book pelvic fractures by circumferential compression. J Orthop Trauma 2002; 16(6): 36773.

[8] Hedrick-Thompson JK. A review of pressure reduction device studies. J Vasc Nurs 1992; 10(4): 3-5. 